\title{
Oxidative Damage and the Prevention of Age-Related Cataracts
}

\author{
David C. Beebe ${ }^{a, b}$ Nancy M. Holekamp ${ }^{a, c}$ Ying-Bo Shui ${ }^{a}$ \\ Departments of a Ophthalmology and Visual Sciences and ${ }^{\mathrm{b} C e l l ~ B i o l o g y}$ and Physiology, Washington University, \\ and 'Barnes Retina Institute, Saint Louis, Mo., USA
}

\section{Key Words}

Oxidative damage $\cdot$ Age-related cataract

\begin{abstract}
Purpose: Cataracts are often considered to be an unavoidable consequence of aging. Oxidative damage is a major cause or consequence of cortical and nuclear cataracts, the most common types of age-related cataracts. Methods: In this review, we consider the different risk factors, natural history and etiology of each of the 3 major types of age-related cataract, as well as the potential sources of oxidative injury to the lens and the mechanisms that protect against these insults. The evidence linking different oxidative stresses to the different types of cataracts is critically evaluated. $\boldsymbol{R e}$ sults: We conclude from this analysis that the evidence for a causal role of oxidation is strong for nuclear, but substantially lower for cortical and posterior subcapsular cataracts. The preponderance of evidence suggests that exposure to increased levels of molecular oxygen accelerates the agerelated opacification of the lens nucleus, leading to nuclear cataract. Factors in the eye that maintain low oxygen partial pressure around the lens are, therefore, important in protecting the lens from nuclear cataract. Conclusions: Maintaining or restoring the low oxygen partial pressure around that lens should decrease or prevent nuclear cataracts.
\end{abstract}

Copyright $\odot 2010$ S. Karger AG, Basel
Cataracts are any opacification of the lens. Although congenital or juvenile cataracts may have serious visual consequences, early-onset cataracts contribute to a relatively small percentage of visual disability. By contrast, age-related cataracts are responsible for nearly half of all blindness worldwide and half of all visual impairment in the USA [1-3]. In the USA alone, surgery for age-related cataracts is the most expensive ocular surgical procedure, with expenditures exceeding USD 3 billion annually [4]. Lens opacities may result from protein aggregation, protein phase separation, or disturbance of the regular alignment or packing of the fiber cells, all of which may lead to increased light scattering. Nuclear opacities sometimes also involve increased coloration, resulting in decreased light transmission. Although cataracts are often considered to be an unavoidable consequence of aging, recent studies of the risk factors associated with human cataracts identified interventions that may prevent cataracts or slow their progression.

\section{The Three Main Types of Age-Related Cataracts}

Age-related lens opacities originate in 1 of 3 regions of the lens: the nucleus, cortex or the posterior pole, just beneath the posterior capsule, resulting in nuclear, cortical or posterior subcapsular cataracts (PSCs), respectively

\section{KARGER}

두 2010 S. Karger AG, Basel

Fax +41613061234

E-Mail karger@karger.ch

www.karger.com
Prof. David C. Beebe, PhD

Department of Ophthalmology and Visual Sciences

Washington University, Campus Box 8096

660 S. Euclid Avenue, Saint Louis, MO 63110 (USA)

E-Mail beebe@wustl.edu 


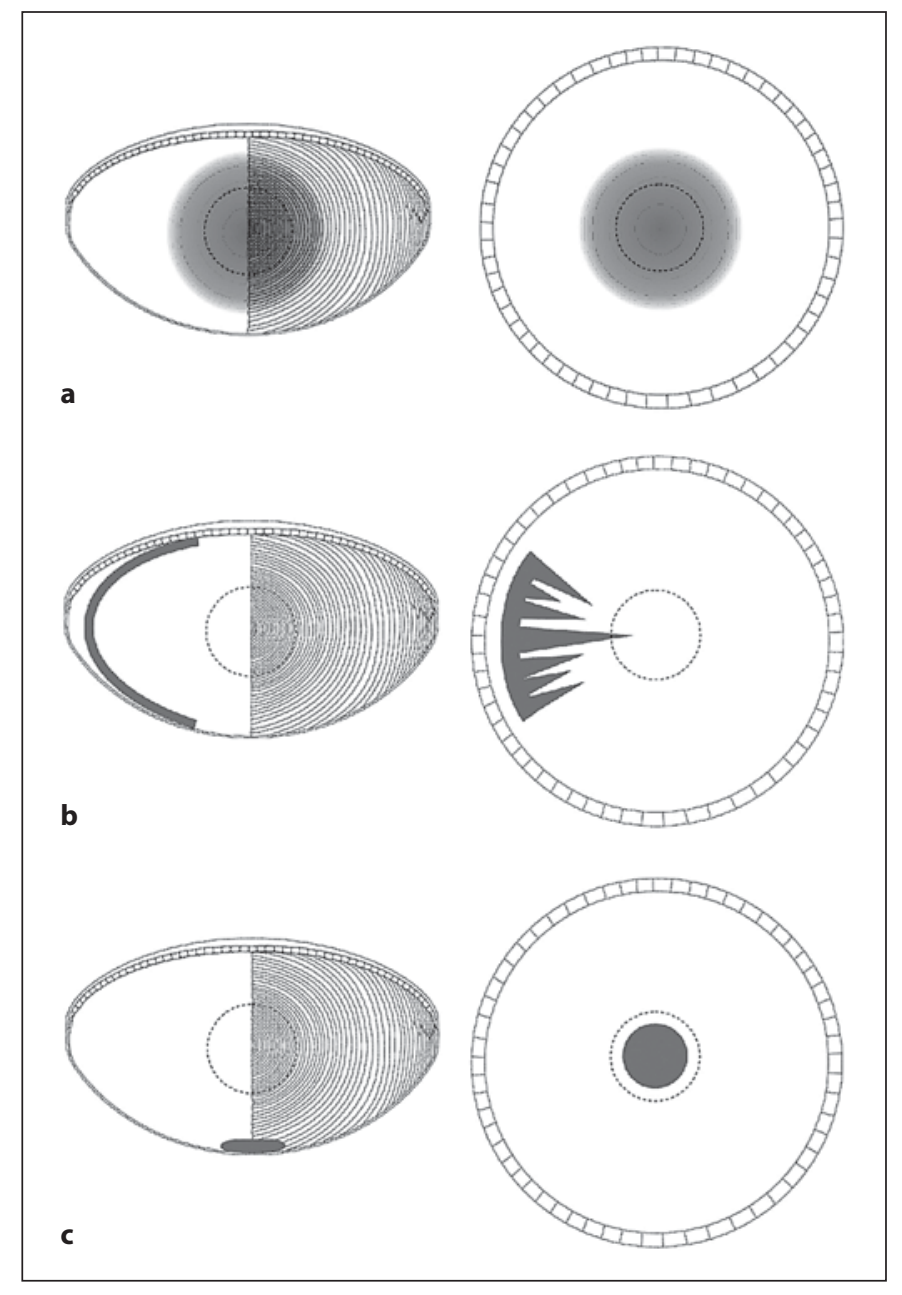

Fig. 1. Diagrams illustrating the 3 main types of age-related cataracts. a Nuclear cataract. A cross-sectional view of the lens is on the left. It shows the location of a nuclear opacity in the central fiber cells. The pattern of fiber cells is illustrated on the right side of this diagram. The right-hand panel in a shows a view of the lens, as seen through the maximally dilated pupil. The dotted circle shows the approximate location of the pupil during maximal constriction. The cataract is in the center of the visual axis. $\mathbf{b}$ Cortical cataract. An opacity in a cluster of cortical fiber cells is illustrated in cross-section on the left and as seen through the pupil on the right. Note that cortical opacities are not visually significant until the opacity reaches the visual axis. c PSC. A cluster of cells has accumulated at the posterior pole of the lens. The opacity is located in the visual axis, where it causes maximal degradation of visual acuity.

(fig. 1). The lens nucleus comprises approximately the central half of the lens and is composed of the fiber cells that were present at birth. Cortical opacities occur in mature (anucleate) fiber cells in the outer half of the lens, cells that were formed postnatally. PSCs form from fiber cells that fail to differentiate properly. Unlike normal fiber cells, these cells do not elongate. Instead, they migrate or are carried along with elongating fiber cells and accumulate at the posterior pole of the lens.

The prevalence of the 3 main types of age-related cataracts differs in different regions of the world and in different racial groups [5]. However, in all areas and populations, PSCs are least common. In northern Japan and northern China, cortical cataracts are more prevalent, while nuclear cataracts predominate in more southern regions of these countries [6]. Similarly, in most tropical areas, nuclear cataracts are most common. Whether these differences are related to differences in genetics, environment, diet or other factors is difficult to discern.

\section{The 'Natural History' and Etiology of Age-Related Cataracts}

Nuclear cataracts are associated with increased light scattering resulting from the aggregation and insolubilization of lens proteins and their increased association with membranes. The causes of increased aggregation are not fully understood, although increased protein oxidation is likely to be involved. During 'normal' aging, the nucleus becomes increasingly stiff, and nuclear light scattering and coloration increase. These changes are associated with increases in insoluble proteins and protein aggregation in all or nearly all persons as they age. The difference between the aging lens nucleus and the increased opacification seen in nuclear cataracts is a matter of degree. In nuclear cataracts the nucleus is substantially harder, more opaque and usually browner than in persons of similar age who are thought of as 'noncataractous'. Clinicians describe them as 'nuclear sclerotic cataracts' as an indication of the increased hardness of the cataractous nucleus.

With increasing age, many types of modifications occur in the abundant crystallin proteins of the lens nucleus. These include protein truncation, cross-linking, denaturation, amino acid racemization, deamidation, glycation and oxidation. The complexity of these changes has made it difficult to determine which might contribute to the age-related hardening and opacification of the nucleus. In contrast to these age-related changes, substantial evidence, collected over more than 50 years, implicates oxidative damage in nuclear cataracts [7].

Most of the events associated with the formation of cortical cataracts are different from those seen in nuclear cataracts. Opacification of the nucleus is not accompa- 
nied by detectable damage to the structure of nuclear fiber cells. By contrast, cortical cataracts are associated with extensive disruption of cell structure and the wholesale precipitation of intracellular proteins. Nuclear cataracts occur uniformly throughout the center of the lens, while cortical cataracts initially involve small clusters of cortical fiber cells. Unlike nuclear opacification, damage to cortical fiber cells is not a necessary consequence of aging. Most individuals never have any evidence of cortical cataracts, while everyone develops some degree of nuclear opacity with age.

The cellular disruption that occurs in cortical cataracts begins near the equator of the lens. Since cortical fiber cells extend from the posterior to the anterior of the lens, this means that opacification begins in the center of a group of fiber cells; the anterior and posterior ends of the same cells remain transparent. Morphological studies found evidence of physical damage early in cortical cataract formation, involving the scission and fragmentation of the cortical fiber cells $[8,9]$. Cortical cataracts progress in severity by the involvement of increasing numbers of adjacent fiber cells and by the extension of the damage from the center of the cells to their anterior and posterior ends. Only when opacification involves the ends of the fiber cells, extending the opacity into the visual axis, does a cortical cataract impair vision.

The cellular changes that lead to the formation of PSCs have been the least studied of all types of cataracts. Examination of postmortem lenses identified streams of cells that appeared to be migrating from the equator to the posterior pole [10-12]. Microscopic examination revealed swollen cells with eosinophilic cytoplasm, suggesting fiber cells that failed to elongate. During the progression of PSCs, increasing numbers of these swollen cells accumulate along the suture planes at the posterior of the lens. Because they lie in the optic axis, these disordered clusters of cells degrade visual acuity. To our knowledge, there have been no biochemical analyses of the contents of human PSCs. Information about their etiology is limited to the risk factors associated with increased risk of developing these opacities. These are described in the following section.

\section{Different Risk Factors for Each Type of Age-Related Cataracts}

Epidemiological studies in populations from around the world identified several factors associated with an increased risk of age-related cataracts [13]. Consistent with their distinct etiologies, the risk profiles for each type of age-related cataract are also different. Based on their different risk factors and pathogenesis, we think that it is appropriate to consider each type of age-related cataract as a different disease, although they all share two common features: increasing prevalence in older individuals and opacification.

Nuclear cataracts are widely associated with poorer diet, lower socioeconomic status, nonprofessional status and lower educational achievement $[2,13]$. The specific risk factors associated with these general socioeconomic categories have been difficult to discern. Clinical trials using vitamin supplementation to lower the risk of cataracts have been unsuccessful or had minimal impact, at least over the typical 5- to 10-year duration of these studies. Some supplements have potentially harmful systemic effects when used at high doses, and 1 recent study reported an increased incidence of cataract surgery in subjects taking vitamin C supplements (but not those taking multivitamin supplements or diets high in vitamin C) [14]. Smoking is a consistent risk factor for nuclear cataracts. The risk of nuclear cataracts increases with the amount and duration of smoking, adding confidence to the existence of a causal relationship between smoking and nuclear opacities. Two studies from diverse ethnic groups identified larger lens size as a risk factor for nuclear cataracts $[15,16]$. Longitudinal studies showed that having a larger lens conferred increased risk of developing nuclear opacities over a 5-year follow-up period [17]. Epidemiological studies have also associated living in a region with increased ambient temperature with increased lens hardening and risk of developing nuclear cataract [6]. Although it may be difficult to establish whether higher ambient temperature is the variable responsible for the higher incidence of nuclear cataracts in these studies, recent laboratory investigations have supported this hypothesis [18]. Finally, studies of twins and examination of familial associations have suggested that about one third of the risk of nuclear cataracts is hereditary, although the genes involved have not been identified $[19,20]$.

High sunlight exposure has been consistently associated with an increased risk of cortical cataracts. The risk of cortical cataract attributable to sunlight is effectively eliminated by wearing a brimmed hat or plastic glasses, making sunlight exposure a preventable risk [21]. In spite of the evidence supporting a role for sunlight in cortical cataracts, the contribution of higher sunlight exposure to the total cataract burden appears to be modest. In most studies, sunlight did not contribute significantly to the 
risk of nuclear or posterior subcapsular opacities. Furthermore, the increased risk of cortical cataract provided by the highest levels of sunlight exposure in a typical population is only about $10 \%$ [22]. A more significant risk of developing cortical cataracts is typically associated with being female, of African heritage and having a family member with cortical cataracts [23-28]. Twin studies and family associations suggest that $50-60 \%$ of the risk of developing cortical cataracts is hereditary. The first human mutations associated with age-related cortical cataracts were recently identified in $E P H 2 A$, a gene encoding a transmembrane tyrosine kinase $[29,30]$. It has not been determined whether it is loss of the kinase activity or the potential adhesive function of the protein that underlies these opacities. As with nuclear cataracts, lens size was identified as a significant risk factor for cortical cataracts. However, unlike nuclear cataracts, the increased prevalence and incidence of cortical opacities were linked to having a smaller, not a larger lens [15-17, 31]. Several studies have shown that diabetics also have an increased risk of developing cortical cataracts and PSCs, although diabetics with well-controlled blood sugar develop a similar spectrum of age-related cataracts as nondiabetics [32, 33].

The major risk factors associated with PSCs are high myopia, diabetes and exposure to therapeutic doses of steroids and ionizing radiation. One study, performed in rats, suggested that high-dose steroids might alter the expression of cell adhesion proteins, like E- and N-cadherin, thereby interfering with fiber cell differentiation [34]. Other than this experimental study, it remains unclear how these diverse factors lead to the defects in fiber cell differentiation that appear to underlie PSCs.

\section{Oxidative Damage and Age-Related Cataracts}

Oxidative damage is a prominent feature of nuclear and cortical cataracts. However, it is important to determine whether oxidation is the cause or the result of cataract formation.

Substantial data suggest that, with increasing age, the lens nucleus becomes more susceptible to oxidation [7]. The cytoplasm of all cells is in a 'reducing environment'. That is, oxidation is inhibited and substances that are capable of being oxidized or reduced are in the reduced state. The tripeptide glutathione is one of the most important reducing agents in the cytoplasm. Glutathione levels are extremely high in peripheral lens fiber cells, where glutathione is synthesized and reduced [35]. Reduced glu- tathione reaches the lens nucleus by diffusion from the surface cells through the abundant network of gap junctions in the lateral membranes of the fiber cells [36, 37]. With increasing age, a greater percentage of the glutathione in the nucleus is found in the oxidized state, as glutathione disulfide or glutathione-protein mixed disulfides. In nuclear cataracts, the proportion of glutathione that is oxidized increases further $[7,35]$. In spite of this, glutathione levels in the outer cortex of the lens remain high in older lenses and in nuclear cataracts. Therefore, the increase in oxidized glutathione in the nucleus is direct evidence of local oxidation during aging and in nuclear cataracts. It also suggests that, with increasing age, the lens nucleus becomes less able to repair oxidative damage.

The factors that determine the extent of glutathione oxidation in the lens nucleus are not known with certainty. However, as the lens ages, it grows larger and its cytoplasm stiffens [38]. This means that the distance that reduced glutathione must diffuse to reach the nucleus increases, while the rate of diffusion through the lens cytoplasm decreases. At the same time, the diffusion of oxidized glutathione from the nucleus back to the lens surface, where it can be reduced, is also impaired. These changes may be sufficient to explain the increased susceptibility of the nucleus to oxidative damage in older lenses.

Cortical cataracts involve the disruption of fiber cell membranes, followed by disintegration of the cytoplasmic contents of the damaged fiber cells. Dissection of lenses with cortical cataracts releases a chalky precipitate from the cataractous areas. This is, presumably, the aggregated remains of the cytoplasmic contents. It seems possible that oxidative damage weakens or stresses the fiber cells, leading to their disruption, or that oxidation is not involved in causing cortical cataracts, but only occurs following loss of membrane integrity. Disruption of the plasma membrane would allow glutathione and other antioxidants to diffuse out of the fiber cells, resulting in the oxidation of the remaining cytoplasmic contents. For these reasons, it is difficult to decide whether cortical cataracts are caused by oxidation or whether oxidation follows the cellular disruption that precedes opacification or some of both. Answers to this conundrum may come from closer examination of the types of oxidative stress that are associated with the formation of age-related cataracts. 


\section{The Potential Causes of Oxidation in Cortical and Nuclear Cataracts}

Of the risk factors identified for cortical cataracts, those that seem most likely to cause increased oxidative damage are high sunlight exposure and diabetes. It is less clear how risk factors like female sex, smaller lens size, African heritage or genetic factors might increase oxidative damage to cortical fiber cells.

Absorption of UV light can generate free radicals, leading to increased oxidative damage $[39,40]$. However, there is an apparent conflict between the exposure of the eye to higher levels of UV radiation and the location of cortical cataracts. Cortical cataracts first appear at the lens equator, the region of the lens that is best protected from exposure to sunlight. This is especially true in bright light, when the pupil is maximally constricted. Similarly, the center of the lens receives the highest dose of UV radiation, yet higher sunlight exposure is not associated with nuclear cataract formation in most studies. Finally, lenses with advanced age-related nuclear color do not show evidence of UV damage, as measured by the destruction of the tryptophan residues in proteins [41]. These observations are difficult to reconcile with the concept that UV light causes cortical cataracts by directly damaging cortical lens fiber cells.

We offer two potential explanations for this paradox. UV light may be absorbed by other eye tissues, the iris, for example, which might produce toxic substances that damage cortical fiber cells [42]. This would be consistent with the increased risk of cataracts associated with dark iris color, seen in some studies [43-45]. However, in 2 of these studies, dark iris color was associated with nuclear cataracts and PSCs, not cortical cataracts.

A second possibility is that higher sunlight exposure increases the stiffness of the lens nucleus, and this increased stiffness contributes to the formation of cortical cataracts. How might this happen? As the lens ages, the nucleus becomes much stiffer, but the cortex remains soft [46]. Stiffening of the nucleus is a major contributor to presbyopia, the nearly universal inability of older individuals to focus on nearby objects [38]. Attempted accommodation by presbyopes would lead to shear forces at the boundary between the soft cortex and the stiff nucleus. If these forces damaged the membranes of cortical fiber cells, cortical cataracts might follow $[9,47]$.

The possibility that physical forces might underlie cortical cataracts is consistent with other risk factors. Having a smaller or thinner lens, a major risk factor for cortical cataracts, might increase the force applied to the lens during accommodation, increasing the risk of cortical damage. Among the many effects of long-term exposure to high blood sugar in diabetes is increased protein glycation, which could increase the stiffness of the nucleus, weaken the cortical fiber cells or both. The mutations in $E P H 2 A$ that are associated with human cortical cataracts could lead to decreased strength of adhesion between fiber cells, increasing the risk of physical damage. Finally, 'mixed' nuclear and cortical cataracts are common, even though the risk factors for each type tend to be distinct. However, since nuclear cataracts are associated with hardening the nucleus, having a nuclear opacity may have the secondary effect of increasing the risk of developing a cortical cataract.

The mechanism by which diabetes damages the lens is complex and has been the subject of much debate. Persons with uncontrolled diabetes often rapidly develop cataracts. These opacities are associated with osmotic damage to the superficial lens cortex that may also involve oxidative damage $[48,49]$. However, uncontrolled diabetes greatly increases mortality, making cataracts the least of the worries for these patients. As stated above, patients with well-controlled diabetes present with a spectrum of cataracts that resemble those seen in the nondiabetic population, albeit at a younger age than nondiabetics [32, 33]. This suggests that the lenses of well-controlled diabetics may be sensitized to the cataract formation, rather than diabetes being a primary cause of opacification.

\section{Is the Lens Subjected to Especially High Levels of Oxidative Stress?}

It is often stated that the lens is subjected to oxidative stress throughout life. Whether this statement is accurate or not, the absence of protein turnover and the limited ability for repair assure that any damage to the mature fiber cells will accumulate over a lifetime. Potential sources of oxidative stress to the lens include UV light, oxidants in the ocular fluids, endogenous oxidants produced in lens cells and smoke constituents.

As stated above, the connection between sunlight exposure and cortical cataracts is likely to be indirect. The iris will block light from reaching more peripheral regions of the lens and most of the shorter, more damaging wavelengths are filtered by the cornea [50]. The absorption of the UV light that does reach the lens over a lifetime does not damage the tryptophan in lens crystallins, even in cataractous lenses, suggesting that the lens is reason- 
ably well protected from the direct effects of light. However, it has been suggested that the increase in UV-absorbing compounds in older lenses ('UV filters') may increasingly sensitize the lens to UV exposure [51]. Finally, the risk of cortical cataract that is attributable to sunlight exposure is quite small. One issue to bear in mind when weighing the epidemiological data: lifetime measures of light exposure have been based on the occupational and recreational exposure of adults. The influence of sunlight exposure in childhood has not been adequately quantified.

Potential damage to the lens from oxidants in the aqueous humor has been a controversial topic. Previous studies suggested that high levels of hydrogen peroxide are present in human aqueous humor [52]. This led to many experiments in which lenses or lens cells were exposed to hydrogen peroxide in vitro. However, the high levels of ascorbate in the aqueous humor interfere with the assay used in this initial study [53]. In addition, aqueous humor produces hydrogen peroxide when exposed to air [54]. Both considerations could lead to overestimation of the hydrogen peroxide levels in the aqueous. More recent studies, which compared the total antioxidant potential of aqueous humor from cataract and glaucoma patients, detected substantially higher antioxidant activity in the cataract patients [55]. The cataract patients also had lower levels of enzymes that are induced by exposure to oxidative stress. This observation suggests that, at least in the cataract patients included in this study, aqueous humor is unlikely to be a source of oxidative stress in cataract.

Several lines of evidence have led to the suggestion that exposure to elevated levels of molecular oxygen can cause nuclear cataracts [56-59]. However, under normal conditions, the level of oxygen around the human lens is very low, protecting the lens from this potential source of damage $[58,60]$. The mechanisms that protect the lens from oxygen and the conditions under which the lens is exposed to higher levels of oxygen are discussed below.

Most cells produce endogenous oxidants as a byproduct of normal metabolism. One potential oxidant is the superoxide anion that is generated in mitochondria during oxidative phosphorylation. This potent reactive oxygen species is converted in the mitochondrion to hydrogen peroxide by superoxide dismutase. The peroxide produced may be metabolized to water by catalase activity, although the disposition of hydrogen peroxide by cells has not been carefully examined. However, lenses produce most of their ATP by glycolysis rather than oxidative phosphorylation [61]. The metabolically active cells of the lens also have very high levels of antioxidants, like glutathione. Therefore, lenses are probably less exposed to and better protected from endogenous oxidants than most other cell types.

One potential source of oxidative stress that is probably important to the lens is smoke. Smoking is one of the most reliable risk factors for nuclear cataracts in epidemiology studies throughout the world $[23,45,62,63]$. Exposure to cooking smoke has also been linked to nuclear cataract [64]. In spite of this, we do not know which components of smoke are harmful to the lens, the mechanisms by which these components cause cataracts or even whether they directly interact with components of the lens. Given the many harmful effects of smoking, better understanding of the mechanism by which smoke causes cataracts is probably not as important as increased support for programs to reduce smoking and exposure to cooking smoke. However, understanding how smoke contributes to cataracts could be important for understanding the etiology of cataract in general.

\section{Oxidation and Nuclear Cataracts}

Clinical and experimental studies strongly implicate increased exposure of the lens to oxygen as a major cause of age-related nuclear cataracts. Patients who were treated daily for more than 1 year with hyperbaric oxygen therapy first developed a myopic shift, then incipient or full-blown nuclear cataracts [56]. A myopic shift is caused by an increase in the refractive power of the lens, presumably due to increased hardening of the lens nucleus. It is commonly a precursor to the formation of age-related nuclear (sclerotic) cataracts. Several subsequent studies of patients undergoing hyperbaric oxygen therapy confirmed the occurrence of a myopic shift, even after relatively short-term therapy $[65,66]$. Importantly, these refractive changes regressed after the discontinuation of short-term therapy, suggesting that the increase in oxidation caused by hyperbaric oxygen is initially reversible. Subsequent studies in experimental animals confirmed the effect of high oxygen exposure on nuclear opacification and provided insight into some of the harmful effects of oxygen on the lens, including increased formation of protein-protein disulfide cross-links [57, 67-70]. These studies provide a direct link between excessive oxygen exposure and nuclear cataract.

Additional support for oxygen exposure in the etiology of nuclear cataracts came from studies of patients undergoing vitrectomy, the destruction and removal of 
the vitreous gel that is typically performed during retinal surgery. Many studies have documented very high rates of nuclear cataract formation (60-95\%) within 2 years after vitrectomy surgery, and vitreoretinal surgeons counsel their patients that they will probably require cataract surgery months to a few years after vitrectomy [71-74]. As mentioned above, oxygen levels near the posterior of the human lens are very low in eyes with an intact vitreous gel (approx. $1 \% \mathrm{O}_{2}$ ). Oxygen levels near the lens increased markedly during vitrectomy and remained significantly elevated for months afterward [58]. Patients with ischemic diabetic retinopathy had significantly lower oxygen in their vitreous and were less likely than nondiabetics to have cataract surgery after vitrectomy $[75,76]$. Our group recently completed an analysis of lens opacification following vitrectomy, using Scheimpflug photography to document the progression of nuclear opacification [Holekamp et al., Am J Ophthalmol, in press]. We found that the lenses of patients with ischemic diabetic retinopathy had lower age-adjusted nuclear opacity at baseline than those of nondiabetics and did not show a significant increase in nuclear opacification after a 1-year follow-up. By contrast, diabetics without ischemic retinopathy and nondiabetics showed rapid progression of nuclear opacity 6 and 12 months after vitrectomy. By 1 year after vitrectomy, $50 \%$ of the nonischemic patients had cataract surgery. These data are consistent with the hypothesis that vitrectomy leads to cataract formation by increasing the exposure of the lens to oxygen.

If exposure of the lens to increased oxygen can cause nuclear cataracts, it is important to understand how the low-oxygen environment around the lens is maintained in eyes that do not undergo vitrectomy. Substantial data suggest that the intact vitreous body protects against nuclear cataract and is essential to maintain the low-oxygen environment at the posterior surface of the lens. It is widely appreciated that the vitreous gel undergoes gradual collapse with age ('vitreous syneresis'). This process begins with the formation of fluid-filled lacunae near the center of the vitreous gel, which progressively coalesce. The collagen fibrils of the vitreous aggregate around the edges of these liquid-filled areas. Eventually, the collapse of the gel and the aggregation of the collagen fibrils create traction on the retina, often leading to the full or partial separation of the remaining vitreous from the retina (posterior vitreous detachment). Although the vitreous liquefies to some degree in all individuals, the extent of loss of gel vitreous can vary greatly between subjects of the same age. Postmortem studies showed that the vitreous of subjects over 50 years ranged from a nearly intact gel to one that was completely or extensively liquefied [77, 78 .

Eyes with more liquefied vitreous are more likely to have nuclear opacities [78]. In subjects between 50 and 70 years old, the state of the vitreous was a better predictor of nuclear opacity than was age. Based on these data, we concluded that the intact gel vitreous preserved the lowoxygen environment around the lens by preventing mixing of the vitreous fluid (fig. 2a). In this view, oxygen diffuses into the vitreous gel from the larger retinal vessels. Microelectrode studies in animals showed that most of this oxygen is consumed by the nearby retina [79]. The consumption of oxygen by the retina maintains an oxygen gradient in the gel near the surface of the retina. However, if the gel is removed, any movements of the head or eye would result in the movement of the vitreous fluid, carrying oxygen away from the retina, mixing it with the vitreous and delivering it to the lens. In this way, the vitreous gel does not inhibit the diffusion of oxygen, but prevents its rapid distribution by fluid mixing (fig. 2b).

In addition to the protection afforded by this physical mechanism, we recently reported that the vitreous consumes oxygen [80]. Actually, it is the ascorbic acid (vita$\min \mathrm{C}$ ) in the vitreous that reacts with oxygen, decreasing the amount of oxygen that reaches the lens. In this reaction, the ascorbate is oxidized to dehydroascorbate, which then spontaneously hydrolyzes, preventing the regeneration of ascorbate by cellular metabolism. The vitreous fluid of subjects with advanced vitreous liquefaction or previous vitrectomy had lower ascorbate and consumed oxygen more slowly than the vitreous of patients with an intact vitreous gel. The reason for the lower ascorbate and decreased oxygen consumption in eyes with no vitreous or mostly liquid vitreous is likely to be due to the increased mixing of the vitreous, which exposes more of the ascorbate to oxygen, thereby lowering ascorbate levels. Thus, the gel state of the vitreous is important to prevent the circulation of oxygen throughout the vitreous chamber and to maintain high levels of ascorbate.

The importance of the vitreous in protecting the lens from oxygen and from nuclear cataract was highlighted by studies in which the vitreous gel was intentionally preserved or destroyed. Surgery to remove preretinal membranes typically involves vitrectomy, which is soon followed by nuclear cataract. However, patients who had a variation of the usual procedure, which did not destroy the vitreous gel, did not develop nuclear cataracts, even after a 5-year follow-up [81]. These studies were important because they showed that nuclear cataract could be prevented, even in patients who had surgery to repair a 

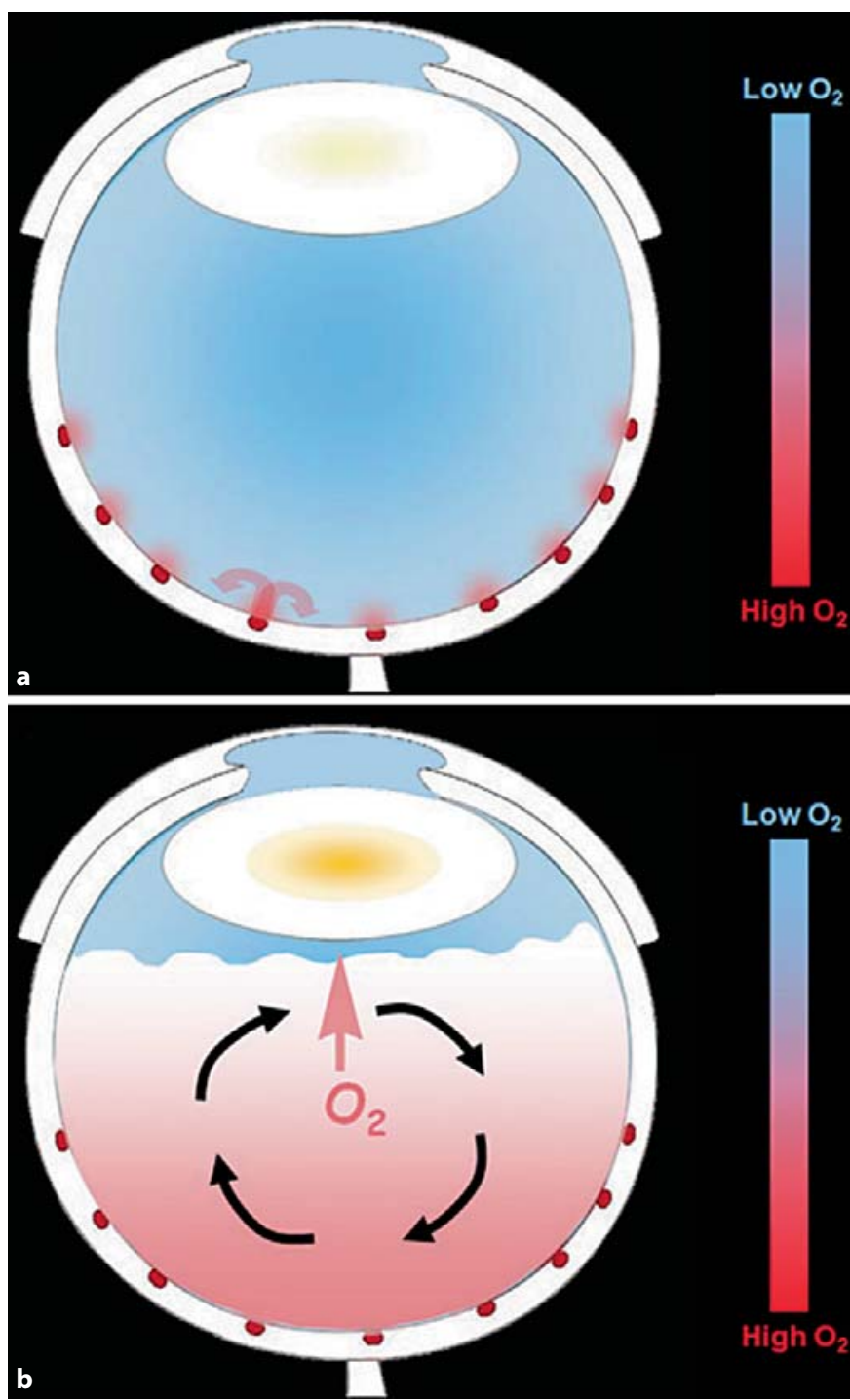

Fig. 2. Diagrams illustrating the distribution of oxygen in the normal eye and following vitrectomy or advanced vitreous degeneration. a An eye with an intact vitreous gel. Oxygen diffuses into the vitreous gel from the large retinal vessels. The curved arrows show that much of this oxygen is subsequently consumed by the adjacent retina. The result is a 'standing gradient' of oxygen near the retina and relatively low oxygen in the midvitreous and near the lens. The lowest oxygen partial pressure is in the midvitreous, due to the consumption of oxygen by the high concentration of ascorbic acid in the intact vitreous. b An eye with advanced vitreous degeneration (posterior vitreous detachment) or after vitrectomy. The posterior of the globe is filled with liquid, instead of gel. This fluid circulates when the eye moves or as a result of convection, as illustrated by the black, curved arrows. When oxygen is carried away from the retina, it can no longer be consumed by retinal metabolism. The increased mixing of oxygen with the ocular fluids delivers more oxygen to the lens (short arrow), where it leads to nuclear opacification. retinal problem, by simply preserving the structure of the vitreous. Unfortunately, patients who had this modified procedure were more likely to have recurrence of the preretinal fibrous membranes, obviating the lens-sparing nature of the modified surgery. Recent studies in animals showed that liquefying the vitreous or creating an enzyme-induced detachment of the vitreous from the retina increased the level of oxygen in the vitreous and resulted in higher levels of oxygen reaching the lens nucleus [82, 83]. These studies demonstrate that the gel state of the vitreous protects the lens from oxygen and from nuclear cataract. The liquefaction of the vitreous, which occurs to a varying extent with age, might be considered to be a slow form of vitrectomy. Loss of the gel state of the vitreous is likely to increase the exposure of the lens to oxygen and place patients at increased risk for nuclear cataract.

\section{Preventing Nuclear Cataract}

The information described above suggests that the gel vitreous decreases the risk of nuclear cataract by protecting the lens from oxygen diffusing from the retinal vasculature. If this is correct, one might prevent nuclear cataracts by preserving the gel state of the vitreous, by replacing the vitreous gel after it is destroyed or degenerates, or by using another method to maintain a low level of oxygen around the lens.

One problem with preserving the gel state of the vitreous is that we have little information about why the vitreous liquefies with age and at a much earlier age in some individuals than in others. Without knowing the cause(s) of vitreous liquefaction, it is difficult to design interventions to preserve the vitreous gel.

Age-related changes have been detected in the fibrillar collagen network of the vitreous gel. Paul Bishop's laboratory showed that collagen IX, which coats the outer surface of the vitreous collagen fibrils, was lost at an exponentially increasing rate from human vitreous, with a half-life of 11 years [84]. Collagen IX has proteoglycan side chains that extend from the surface of the vitreous fibrils and may prevent the collapse of the collagen network. Concurrently, more of the core collagen II in the vitreous fibrils could be recognized by antibody binding. These data suggest that, with increasing age, the loss of collagen IX will lead to an increasing tendency of the fibrils of the vitreous gel to aggregate, eventually promoting the collapse of the gel. However, these changes occurred on a similar time scale in all samples, yet vitreous liquefaction occurs at quite different rates in different in- 
dividuals $[77,78]$. Therefore, it is possible that the degradation or modification of one or more additional components of the vitreous contributes to liquefaction.

Patients with mutations in COL2A1, COL9A1 and COL11A1 or COL11A2, the fibrillar collagens that make up the backbone of the vitreous gel, have Stickler syndrome, resulting in no or little vitreous gel from an early age (patients with mutations in COL11A1 have also been categorized as having Marshall syndrome, a related syndrome). Patients with Wagner syndrome, resulting from mutations in CSPG2, a chondroitin sulfate proteoglycan, also called versican, also have early vitreous degeneration, frequent retinal detachment and an increased risk of cataract. Early vitreous liquefaction in patients with mutations in the genes that encode these abundant components of the vitreous raises the possibility that minor variations in these genes or other structural components of the vitreous could lead to reduced stability of the vitreous gel, leading to early vitreous degeneration in some individuals. If, as suggested above, early vitreous degeneration increases the risk of nuclear cataract, variations in these genes could account for some or all of the risk of nuclear cataracts that is attributable to heredity. Since no genetic risk factors have been identified for nuclear cataracts, this possibility remains to be tested.

One of the major limitations in studying the factors that influence vitreous liquefaction is the lack of a simple, reliable, noninvasive measure of the gel state of the vitreous. Lack of such a test makes genetic and epidemiological studies of vitreous liquefaction difficult. One published method used ultrasound to quantify relatively vitreous liquefaction, but, to our knowledge, this approach has not yet been independently validated or used to screen for vitreous liquefaction [85]. If such a test were validated and easily used in the clinic, it could be used to test the association between vitreous degeneration and nuclear cataract and to identify genetic and environmental causes of vitreous liquefaction.

Several groups have developed synthetic gels to replace the vitreous after vitrectomy [86-89]. In most cases, these preparations were designed to assist in re-attaching the retina after detachment. To our knowledge, the ability of these gels to restore the low oxygen levels near the posterior surface of the lens has not been tested.

The foregoing section illustrates several opportunities to test the hypothesis that loss of the gel state of the vitreous and increased exposure of the lens to oxygen result in nuclear cataract formation. If this hypothesis were validated in human subjects, practical interventions to prevent nuclear cataracts could follow. Since vitrectomy surgery is a common procedure that causes rapid and reliable opacification of the lens nucleus, patients undergoing vitrectomy offer a promising 'model system' to test this hypothesis. Success in protecting postvitrectomy patients from developing nuclear cataracts would lay the groundwork for interventions to prevent these cataracts in subjects with advanced vitreous degeneration.

\section{Acknowledgements}

Grant funding that supported this research was provided by NIH grants EY04853 and EY15863, core grant EY02687, the BRI Research Fund and an unrestricted grant to the Department of Ophthalmology and Visual Sciences from Research to Prevent Blindness.

\section{References}

1 The Eye Diseases Prevalence Research Group: Causes and prevalence of visual impairment among adults in the United States. Arch Ophthalmol 2004;122:477-485.

2 West S: Epidemiology of cataract: accomplishments over 25 years and future directions. Ophthalmic Epidemiol 2007;14:173178.

3 Resnikoff S, et al: Global data on visual impairment in the year 2002. Bull World Health Organ 2004;82:844-851.

4 Salm M, Belsky D, Sloan FA: Trends in cost of major eye diseases to Medicare, 1991 to 2000. Am J Ophthalmol 2006;142:976-982.

Preventing Age-Related Cataracts
5 Sasaki K, et al: Racial differences of lens transparency properties with aging and prevalence of age-related cataract applying a WHO classification system. Ophthalmic Res 2004;36:332-340.

6 Sasaki H, et al: High prevalence of nuclear cataract in the population of tropical and subtropical areas. Dev Ophthalmol 2002;35: 60-69.

-7 Truscott RJW: Age-related nuclear cataract - oxidation is the key. Exp Eye Res 2004;80: 709-725.

8 Brown NP, et al: Is cortical spoke cataract due to lens fibre breaks? The relationship between fibre folds, fibre breaks, waterclefts and spoke cataract. Eye 1993;7:672-679.
9 Michael R, et al: Morphology of age-related cuneiform cortical cataracts: the case for mechanical stress. Vision Res 2008;48:626-634.

10 Eshaghian J, Streeten BW: Human posterior subcapsular cataract: an ultrastructural study of the posteriorly migrating cells. Arch Ophthalmol 1980;98:134-143.

-11 Eshagian J: Human posterior subcapsular cataracts. Trans Ophthalmol Soc UK 1982; 102:364-368

12 Streeten BW, Eshaghian J: Human posterior subcapsular cataract: a gross and flat preparation study. Arch Ophthalmol 1978;96: 1653-1658. 
13 Beebe D: Lens; in Kaufman PL, Alm A (eds): Adler's Physiology of the Eye. St Louis, Mosby, 2003, pp 117-158.

14 Rautiainen S, et al: Vitamin C supplements and the risk of age-related cataract: a population-based prospective cohort study in women. Am J Clin Nutr 2009, DOI: ajcn.2009.28528

15 Praveen MR, et al: Lens thickness of Indian eyes: impact of isolated lens opacity, age, axial length, and influence on anterior chamber depth. Eye 2009;23:1542-1548.

16 Klein B, Klein R, Moss S: Correlates of lens thickness: the Beaver Dam Eye Study. Invest Ophthalmol Vis Sci 1998;39:1507-1510.

$\checkmark 17$ Klein BE, Klein R, Moss SE: Lens thickness and five-year cumulative incidence of cataracts: the Beaver Dam Eye Study. Ophthalmic Epidemiol 2000;7:243-248.

18 Heys KR, Friedrich MG, Truscott RJ: Presbyopia and heat: changes associated with aging of the human lens suggest a functional role for the small heat shock protein, alpha-crystallin, in maintaining lens flexibility. Aging Cell 2007;6:807-815.

19 Hammond CJ, et al: Genetic and environmental factors in age-related nuclear cataracts in monozygotic and dizygotic twins. $\mathrm{N}$ Engl J Med 2000;342:1786-1790.

20 Heiba IM, et al: Genetic etiology of nuclear cataract: evidence for a major gene. Am J Med Genet 1993;47:1208-1214.

$\checkmark 21$ Taylor HR, et al: Effect of ultraviolet radiation on cataract formation. N Engl J Med 1988;319:1429-1433.

22 West SK, et al: Sunlight exposure and risk of lens opacities in a population-based study: the Salisbury Eye Evaluation Project. JAMA 1998;280:714-718.

-23 McCarty CA, Nanjan MB, Taylor HR: Attributable risk estimates for cataract to prioritize medical and public health action. Invest Ophthalmol Vis Sci 2000;41:3720-3725.

24 Leske MC, et al: Prevalence of lens opacities in the Barbados Eye Study. Arch Ophthalmol 1997;115:105-111.

-25 Risk factors for age-related cortical, nuclear, and posterior subcapsular cataracts. The Italian-American Cataract Study Group. Am J Epidemiol 1991;133:541-553.

26 Hammond CJ, et al: The heritability of agerelated cortical cataract: the Twin Eye Study. Invest Ophthalmol Vis Sci 2001;42:601-605.

27 Leske MC, Chylack LT Jr, Wu SY: The Lens Opacities Case-Control Study: risk factors for cataract. Arch Ophthalmol 1991;109: 244-251.

28 Congdon N, et al: Cortical, but not posterior subcapsular, cataract shows significant familial aggregation in an older population after adjustment for possible shared environmental factors. Ophthalmology 2005;112: 73-77.

29 Jun G, et al: EPHA2 is associated with agerelated cortical cataract in mice and humans. PLoS Genet 2009;5:e1000584. 30 Shiels A, et al: The EPHA2 gene is associated
with cataracts linked to chromosome $1 \mathrm{p}$. Mol Vis 2008;14:2042-2055.

31 Wong TY, et al: Refractive errors, axial ocular dimensions, and age-related cataracts: the Tanjong Pagar Survey. Invest Ophthalmol Vis Sci 2003;44:1479-1485.

32 Oishi N, et al: Correlation between adult diabetic cataracts and red blood cell aldose reductase levels. Invest Ophthalmol Vis Sci 2006;47:2061-2064.

33 Bron AJ, et al: The lens in diabetes. Eye 1993; 7:260-275.

34 Lyu J, et al: Alteration of cadherin in dexamethasone-induced cataract organ-cultured rat lens. Invest Ophthalmol Vis Sci 2003;44: 2034-2040.

35 Giblin FJ: Glutathione: a vital lens antioxidant. J Ocul Pharmacol Ther 2000;16:121135.

36 Sweeney MHJ, Garland DL, Truscott RJW: Movement of cysteine in intact monkey lenses: the major site of entry is the germinative region. Exp Eye Res 2003;77:245-251.

-37 Sweeney MH, Truscott RJ: An impediment to glutathione diffusion in older normal human lenses: a possible precondition for nuclear cataract. Exp Eye Res 1998;67:587-595.

38 McGinty SJ, Truscott RJ: Presbyopia: the first stage of nuclear cataract? Ophthalmic Res 2006;38:137-148.

39 Truscott RJW: Human cataract: the mechanisms responsible; light and butterfly eyes. Int J Biochem Cell Biol 2003;35:1500-1504.

40 Wood AM, Truscott RJ: UV filters in human lenses: tryptophan catabolism. Exp Eye Res 1993;56:317-325.

41 Harding JJ: The untenability of the sunlight hypothesis of cataractogenesis. Doc Ophthalmol 1994;88:345-349.

42 Andley UP, et al: The role of prostaglandins $\mathrm{E}_{2}$ and $\mathrm{F}_{2} \alpha$ in ultraviolet radiation- induced cortical cataracts in vivo. Invest Ophthalmol Vis Sci 1996;37:1539-1548.

43 Cumming RG, Mitchell P, Lim R: Iris color and cataract: the Blue Mountains Eye Study. Am J Ophthalmol 2000;130:237-238.

44 Younan C, et al: Iris color and incident cataract and cataract surgery: the Blue Mountains Eye Study. Am J Ophthalmol 2002;134: 273-274

45 AREDS: Risk factors associated with age-related nuclear and cortical cataract: a casecontrol study in the Age-Related Eye Disease Study, AREDS report No 5. Ophthalmology 2001;108:1400-1408.

46 Heys KR, Cram SL, Truscott RJ: Massive increase in the stiffness of the human lens nucleus with age: the basis for presbyopia? Mol Vis 2004; 10:956-963.

47 Pau H: Cortical and subcapsular cataracts: significance of physical forces. Ophthalmologica 2006;220:1-5.

48 Hegde KR, Varma SD: Combination of glycemic and oxidative stress in lens: implications in augmentation of cataract formation in diabetes. Free Radic Res 2005;39:513-517.
49 Obrosova IG, Fathallah L, Lang HJ: Interaction between osmotic and oxidative stress in diabetic precataractous lens: studies with a sorbitol dehydrogenase inhibitor. Biochem Pharmacol 1999;58:1945-1954.

50 Dillon J, et al: The optical properties of the anterior segment of the eye: implications for cortical cataract. Exp Eye Res 1999;68:785795.

51 Mizdrak J, et al: Tryptophan-derived ultraviolet filter compounds covalently bound to lens proteins are photosensitizers of oxidative damage. Free Radic Biol Med 2008;44: 1108-1119.

52 Garner MH, et al: Biochemical evidence for membrane disintegration in human cataracts. Proc Natl Acad Sci USA 1981;78:18921895.

53 Garcia-Castineiras S, et al: Aqueous humor hydrogen peroxide analysis with dichlorophenol-indophenol. Exp Eye Res 1992;55:919.

54 Spector A, Ma W, Wang RR: The aqueous humor is capable of generating and degrading $\mathrm{H}_{2} \mathrm{O}_{2}$. Invest Ophthalmol Vis Sci 1998; 39:1188-1197.

55 Ferreira SM, et al: Oxidative stress markers in aqueous humor of glaucoma patients. Am J Ophthalmol 2004;137:62-69.

56 Palmquist BM, Philipson B, Barr PO: Nuclear cataract and myopia during hyperbaric oxygen therapy. Br J Ophthalmol 1984;68: 113-117.

57 Giblin FJ, et al: Nuclear light scattering, disulfide formation and membrane damage in lenses of older guinea pigs treated with hyperbaric oxygen. Exp Eye Res 1995;60:219235

58 Holekamp NM, Shui YB, Beebe DC: Vitrectomy surgery increases oxygen exposure to the lens: a possible mechanism for nuclear cataract formation. Am J Ophthalmol 2005; 139:302-310

59 Beebe DC: Maintaining transparency: a review of the developmental physiology and pathophysiology of two avascular tissues. Semin Cell Dev Biol 2008:19:125-133.

60 Helbig H, et al: Oxygen in the anterior chamber of the human eye. Ger J Ophthalmol 1993;2:161-164.

-61 Winkler BS, Riley MV: Relative contributions of epithelial cells and fibers to rabbit lens ATP content and glycolysis. Invest Ophthalmol Vis Sci 1991;32:2593-2598.

-62 Cumming RG, Mitchell P: Alcohol, smoking, and cataracts: the Blue Mountains Eye Study. Arch Ophthalmol 1997;115:12961303.

63 Hodge WG, Whitcher JP, Satariano W: Risk factors for age-related cataracts. Epidemiol Rev 1995;17:336-346.

64 Pokhrel AK, et al: Case-control study of indoor cooking smoke exposure and cataract in Nepal and India. Int J Epidemiol 2005;34: 702-708. 
-65 Evanger K, et al: Ocular refractive changes in patients receiving hyperbaric oxygen administered by oronasal mask or hood. Acta Ophthalmol Scand 2004;82:449-453.

66 Fledelius HC, Jansen EC, Thorn J: Refractive change during hyperbaric oxygen therapy. a clinical trial including ultrasound oculometry. Acta Ophthalmol Scand 2002;80:188190.

67 Bantseev V, et al: Effect of hyperbaric oxygen on guinea pig lens optical quality and on the refractive state of the eye. Exp Eye Res 2004; 78:925-931

68 Gosselin ME, et al: Raman spectroscopic evidence for nuclear disulfide in isolated lenses of hyperbaric oxygen-treated guinea pigs. Exp Eye Res 2007;84:493-499.

69 Padgaonkar VA, et al: Hyperbaric oxygen in vivo accelerates the loss of cytoskeletal proteins and MIP26 in guinea pig lens nucleus. Exp Eye Res 1999;68:493-504.

70 Simpanya MF, et al: Aggregation of lens crystallins in an in vivo hyperbaric oxygen guinea pig model of nuclear cataract: dynamic light-scattering and HPLC analysis. Invest Ophthalmol Vis Sci 2005;46:4641-4651.

71 Cherfan GM, et al: Nuclear sclerotic cataract after vitrectomy for idiopathic epiretinal membranes causing macular pucker. Am J Ophthalmol 1991;111:434-438.
72 De Bustros S, et al: Nuclear sclerosis after vitrectomy for idiopathic epiretinal membranes. Am J Ophthalmol 1988;105:160-164.

73 Thompson JT, et al: Progression of nuclear sclerosis and long-term visual results of vitrectomy with transforming growth factor beta-2 for macular holes. Am J Ophthalmol 1995;119:48-54

74 Van Effenterre G, et al: Is vitrectomy cataractogenic? Study of changes of the crystalline lens after surgery of retinal detachment. J Fr Ophtalmol 1992;15:449-454.

75 Holekamp NM, Shui Y-B, Beebe D: Lower intraocular oxygen tension in diabetic patients: possible contribution to decreased incidence of nuclear sclerotic cataract. Am J Ophthalmol 2006;141:1027-1032.

76 Smiddy WE, Feuer W: Incidence of cataract extraction after diabetic vitrectomy. Retina 2004;24:574-581.

77 O’Malley P: The pattern of vitreous syneresis: aA study of 800 autopsy eyes; inIrvine AR, O'Malley C (eds): Advances in Vitreous Surgery. Springfield, Thomas, 1976, pp 1733.

78 Harocopos GJ, et al: Importance of vitreous liquefaction in age-related cataract. Invest Ophthalmol Vis Sci 2004;45:77-85.

79 Alder VA, Cringle SJ: Vitreal and retinal oxygenation. Graefes Arch Clin Exp Ophthalmol 1990;228:151-157.

-80 Shui Y-B, et al: The gel state of the vitreous and ascorbate-dependent oxygen consumption: relationship to the etiology of nuclear cataracts. Arch Ophthalmol 2009;127:1-8.
81 Sawa M, et al: Nonvitrectomizing vitreous surgery for epiretinal membrane: long-term follow-up. Ophthalmology 2005;112:14021408

82 Quiram PA, et al: Microplasmin-induced posterior vitreous detachment affects vitreous oxygen levels. Retina 2007;27:10901096

83 Giblin FJ, et al: Enzyme-induced posterior vitreous detachment in the rat produces increased lens nuclear $\mathrm{pO}_{2}$ levels. Exp Eye Res 2009;88:286-292.

84 Bishop PN, et al: Age-related changes on the surface of vitreous collagen fibrils. Invest Ophthalmol Vis Sci 2004;45:1041-1046.

85 Walton KA, et al: Age-related changes in vitreous mobility as measured by video B scan ultrasound. Exp Eye Res 2002;74:173-180.

$>86$ Swindle-Reilly KE, et al: Rabbit study of an in situ forming hydrogel vitreous substitute. Invest Ophthalmol Vis Sci 2009;50:48404846.

87 Maruoka S, et al: Biocompatibility of polyvinylalcohol gel as a vitreous substitute. Curr Eye Res 2006;31:599-606.

88 Soman N, Banerjee R: Artificial vitreous replacements. Biomed Mater Eng 2003;13:5974.

89 Suri S, Banerjee R: In vitro evaluation of in situ gels as short term vitreous substitutes. J Biomed Mater Res A 2006;79:650-664. 